\title{
The detection of white matter alterations in obsessive-compulsive disorder revealed by TRActs Constrained by UnderLying Anatomy (TRACULA)
}

This article was published in the following Dove Press journal: Neuropsychiatric Disease and Treatment

\author{
Anri Watanabe \\ Takashi Nakamae' \\ Yuki Sakai ${ }^{2}$ \\ Seiji Nishida' \\ Yoshinari Abe' \\ Kei Yamada ${ }^{3}$ \\ Isao Yokota ${ }^{4}$ \\ Jin Narumoto' \\ 'Department of Psychiatry, Graduate \\ School of Medical Science, Kyoto \\ Prefectural University of Medicine, \\ Kyoto, Japan; ${ }^{2}$ Department of Neural \\ Computation for Decision-Making, \\ Advanced Telecommunications \\ Research Institute International \\ Brain Information Communication \\ Research Laboratory Group, Kyoto, \\ Japan; ${ }^{3}$ Department of Radiology, \\ Graduate School of Medical Science, \\ Kyoto Prefectural University of \\ Medicine, Kyoto, Japan; ${ }^{4}$ Department \\ of Biostatistics, Graduate School of \\ Medical Science, Kyoto Prefectural \\ University of Medicine, Kyoto, Japan
}

Correspondence: Takashi Nakamae Kajii-cho, Kawaramachi-Hirokoji, Kamigyo-ku, Kyoto 602-8566, Japan $\mathrm{Tel}+8|7525| 5612$

Fax $+8 \mid 75$ 25। 5839

Email nakamae@koto.kpu-m.ac.jp
Purpose: In recent years, a large number of studies have investigated obsessive-compulsive disorder (OCD) using diffusion-weighted images (DWIs) and have reported microstructural abnormalities in various regions, mainly the corpus callosum and cingulum. In the present study, we aimed to detect microstructural changes in the white matter using whole-brain tractography.

Patients and methods: We obtained MRI data from 25 adult non-medicated OCD patients and 27 healthy controls. DWI data from MRI scans were analyzed by the automated probabilistic tractography method referred to as TRActs Constrained by UnderLying Anatomy (TRACULA). We investigated diffusivity parameters: fractional anisotropy, radial diffusivity (RD), axial diffusivity, and mean diffusivity in 18 major white matter tracts and examined indices to know which measurements in which fibers can predict the diagnosis of OCD.

Results: Compared to healthy controls, OCD patients had significantly increased RD in the forceps major and a reduction of RD in the right cingulum angular (infracallosal) bundle. There was no correlation between these values and the clinical features.

Conclusion: We found RD alterations in the forceps major and the right cingulum angular (infracallosal) bundle, which might be associated with myelination changes in the temporal and occipital regions in OCD. Our results suggest that the pathogenesis of OCD may include abonormality of myelination status in not only the fronto-striato-thalamic circuit but also the posterior and temporal regions.

Keywords: tractography, radial diffusivity, forceps major, cingulum

\section{Introduction}

Obsessive-compulsive disorder (OCD) is characterized by recurrent thoughts and repetitive behaviors that lead to social functional impairment. Lifetime prevalence of OCD has been estimated at 2.3\%. ${ }^{1}$ Besides, individuals with OCD experience chronic and severe course. OCD is among the 20 leading causes of years-of-life lost due to disability, ${ }^{2}$ and therefore has serious effects on individual quality of life and society. ${ }^{3}$

In the last few decades, a large number of studies of OCD have detected structural and functional alterations in the brain. Since previous studies have reported elevated metabolic rates in heads of the caudate nuclei and orbital gyri using positron emission tomography, ${ }^{4}$ as well as decreased orbitofrontal cortex volume in patients with OCD,${ }^{5}$ it has been hypothesized that the orbitofrontal cortex and the ventral striatum are involved in the neuropathology of OCD. A meta-analysis of functional MRI 
studies identified abnormalities in the cortico-striato-thalamic circuits, ${ }^{6}$ and a systematic review and meta-analysis of diffusion tensor imaging (DTI) studies also highlighted the cortico-striato-thalamic circuit. ${ }^{7}$ Consequently, this circuit is considered to underpin the pathogenesis of OCD. However, in recent years, besides this cortico-striato-thalamic circuit, alterations in other foci including dorsolateral/medial prefrontal cortex, temporal, parietal and occipital regions, anterior insula, and internal capsule have been detected, ${ }^{8,9}$ suggesting widespread abnormalities are probably involved in OCD pathology. Therefore, there is a need to examine the whole brain of OCD patients.

As genetic studies of OCD have previously reported specific genes related to myelin, ${ }^{10-12}$ there is the possibility that white matter alteration may be related to the pathology of OCD. Numerous studies have reported changes of white matter in the corpus callosum, ${ }^{13-22}$ cingulum bundle, ${ }^{15,17-20,23-27}$ and parietal regions. ${ }^{6,15,23,28}$ A meta-analysis revealed white matter abnormalities mainly in crossing between the anterior body of the corpus callosum and cingulum and other various regions. ${ }^{29}$ And also, previous researches showed their results of diffusivity measurements were opposite even in the same tract (eg, the body of corpus callosum ${ }^{16-18}$ and the internal capsule $^{18,30}$ ) and the results from OCD patients were inconsistent. Until now, most DTI studies have used voxel-based morphometric ${ }^{13,17,25,28}$ analysis and tract-based spatial statistics (TBSS). ${ }^{16,18-20,22,30,31}$ These methods can detect voxels with abnormalities but do not reveal the specific white matter bundles harboring the abnormalities. In contrast, tractography identifies specific white matter bundles with abnormalities, which enables the determination of brain regions that are connected by these fibers. ${ }^{32}$

TRActs Constrained by UnderLying Anatomy (TRACULA) is one of the tractography methods whose advantage is sensitive to the specific white matter tract which we target. ${ }^{33}$ Therefore, it allows identification of the specific tracts with white matter alterations. Previous studies focused on the corpus callosum, ${ }^{21,34}$ and fronto-striatal fibers ${ }^{35}$ have used tractography to investigate OCD pathology. However, there have been few tractographic studies that have investigated multiple major white matter bundles. TRACULA conducts whole-brain tractography in the native diffusion space and makes it possible to reconstruct 18 major white matter fibers. TRACULA utilizes prior information of the anatomy obtained from a set of trained subjects where the tracts are labeled manually. The prior information is the probabilities that the tracts pass through or to the left, right, anterior, posterior, superior, and inferior of a certain label determined by FreeSurfer software, and is validated by the accuracy of automated segmentation of the T1-weighted images (T1WIs). ${ }^{36}$ TRACULA does not use deterministic region of interest (ROI) but uses prior probabilities from a set of training subjects. It models the likelihood of the anatomical neighborhood of a tract how likely each tract is to go through or next to each of the labels of the FreeSurfer subcortical segmentation and cortical parcellation. The other automatic tractography method, for instance, that is named as Automated Fiber Quantification defines two waypoint ROIs and reconstructs the pathways which go through ROIs. ${ }^{37}$ TRACULA adopts the ball-and-stick model, which sets multiple anisotropic compartments per voxel and global tractography that is not sensitive to areas of high local uncertainty. Therefore, this tractographic method, TRACULA, enables estimation of even areas of low anisotropy and tract crossing. In other words, an advantage of TRACULA is its ability to identify the brain region with alteration as a part of the tracts, and has little effects by fiber junctions or crossings. In this study, we aimed to comprehensively investigate alterations in white matters in OCD patients by exploratory analysis using TRACULA in order to detect diffusivity measurements in white matter tracts that could predict the diagnosis of OCD.

\section{Patients and methods Participants}

Twenty-seven patients with OCD and 28 age-matched healthy controls (HCs) were scanned with MRI and two patients and one control were excluded because of their inadequate reconstruction. The subjects were 25 adult patients diagnosed with OCD and 27 age-matched HC. Patients were recruited from University Hospital, Kyoto Prefectural University of Medicine, Kyoto, Japan. All patients were diagnosed with OCD using the Structured Clinical Interview for DSM-IV Axis I Disorders Patient Edition (SCID-I/P). ${ }^{38}$ All patients had a sole diagnosis of OCD and none had taken psychotropic medications for at least 8 weeks. Five OCD patients were medication-naive and 20 patients had psychotropic medication histories. Obsessive-compulsive symptom severity was quantified by the Yale-Brown Obsessive Compulsive Scale (Y-BOCS). ${ }^{39}$ We also assessed the excess of depression and anxiety by Hamilton Depression Rating Scale (HAM-D) and Hamilton Anxiety Rating Scale (HAM-A) on the OCD patients. Patients had no current comorbidities which occurred frequently in OCD (eg, major depressive disorder) and only two patients had a previous diagnosis of major depressive disorder.

Exclusion criteria were as follows: 1) significant disease such as neurological diseases and disorders of the pulmonary, 
cardiac, renal, hepatic, endocrine, or metabolic systems; 2) current or past DSM-IV axis I diagnosis of any psychiatric disorders except OCD; and 3) DSM-IV diagnosis of mental retardation and pervasive developmental disorders based on a clinical interview and psychological history.

There were no histories of psychiatric disorders in $\mathrm{HC}$, as determined by the Structured Clinical Interview for DSM-IV Axis I Disorders/Non-patient Edition (SCID-I/NP).

Kyoto-Prefectural University of Medicine Research Ethics Committee approved this study and all participants gave written informed consent.

\section{MRI acquisition}

Participants underwent MRI scanning using a whole-body 3-T MR scanner (Achieva 3.0T T; Philips Medical Systems, Best, the Netherlands) with an eight-channel phasedarray head coil to obtain T1WI and diffusion-weighted images (DWIs).

We acquired T1-weighted 3-dimensional magnetizationprepared rapid gradient-echo (3D-MPRAGE) sequences: repetition time, $7.1 \mathrm{~ms}$; echo time, $3.3 \mathrm{~ms}$; flip angle, $10^{\circ}$; field of view, $256 \mathrm{~mm}$; acquisition matrix, 256×256×170 slices; section thickness, $1.0 \mathrm{~mm}$; and voxel size, $1.0 \times 1.0 \times 1.0 \mathrm{~mm}$. Then, whole-brain DWIs were obtained using a single-shot spinecho echo-planar sequence with 32 Stejskal-Tanner motionprobing gradient orientations. The scanning parameters were repetition time, $7,181 \mathrm{~ms}$; echo time, $58 \mathrm{~ms} ; b=1,000 \mathrm{~s} / \mathrm{mm}^{2}$; flip angle, $90^{\circ}$; field of view, $224 \times 224 \times 120 \mathrm{~mm}^{3}$; acquisition matrix, $112 \times 110$; slice thickness, $2.0 \mathrm{~mm}$; axial slices, 60; and voxel size, $2.0 \times 2.0 \times 2.0 \mathrm{~mm}^{3}$. One image without motion probing gradient $\left(b=0 \mathrm{~s} / \mathrm{mm}^{2}\right)$ and 32 images with it $\left(b=1,000 \mathrm{~s} / \mathrm{mm}^{2}\right)$ were obtained for each subject.

\section{$\mathrm{MRI}$ analysis}

T1WIs were processed using FreeSurfer stable version 5.3.0 (http://surfer.nmr.mgh.harvard.edu) and surface-based cortical reconstruction was performed. This reconstruction includes affine transformation from the original volume to MNI305, subcortical segmentation based on probabilistic information about the location of structures and cortical parcellation which assigns an anatomical label to each location on a cortical surface model based on probabilistic information using the "Desikan-Killiany" cortical atlas. ${ }^{40}$ After subcortical segmentation and cortical parcellation, we examined the axial, sagittal, and coronal views and found there were neither too much removed nor not enough removed areas. Consequently, 18 major white-matter pathways were automatically reconstructed with TRACULA ${ }^{33}$ by DWIs using FreeSurfer stable version 5.3.0. We also checked tensor orientations by representing directions as lines and found no misalignment. Preprocessing steps include eddy-current compensation, computation of measures of head motion, intra-subject registration (from individual DWI to individual T1WI), inter-subject registration (from T1WI to the FreeSurfer common template space), cortical and white matter mask creation, tensor fitting for the extraction of tensor-based measures, and the computation of anatomical priors for white matter pathways from the TRACULA atlas. TRACULA computed the measures of head motion in the DWIs of participants, ${ }^{41}$ which enabled the average translational distance and degree of rotation to be compared between groups. There was no significant difference in head motion measures between OCD and HC groups $(p<0.05)$, hence we ignored spurious group differences. The next step was to fit a ball-and-stick model, which is one of the diffusion model to the DWIs using FMRIB Software Library's bedpost. ${ }^{42}$ The final step was to reconstruct 18 major white matter pathways by using each probability distribution and fitting the shape of each pathway to the ball-and-stick model and the pathway priors. We obtained the estimated distribution threshold at $20 \%$ of maximum. ${ }^{33}$ The 18 reconstructed white matter pathways included the corticospinal tract, inferior longitudinal fasciculus, uncinate fasciculus, anterior thalamic radiation, cingulum cingulate gyrus (supracallosal) bundle, cingulum angular (infracallosal) bundle (CAB), superior longitudinal fasciculus parietal bundle, superior longitudinal fasciculus temporal bundle, corpus callosum forceps major (FMaj), and corpus callosum forceps minor (Figure 1) and scalar output volumes of the tensor fits were fractional anisotropy (FA), radial diffusivity (RD), axial diffusivity (AD), and mean diffusivity (MD). We checked all reconstructed pathways
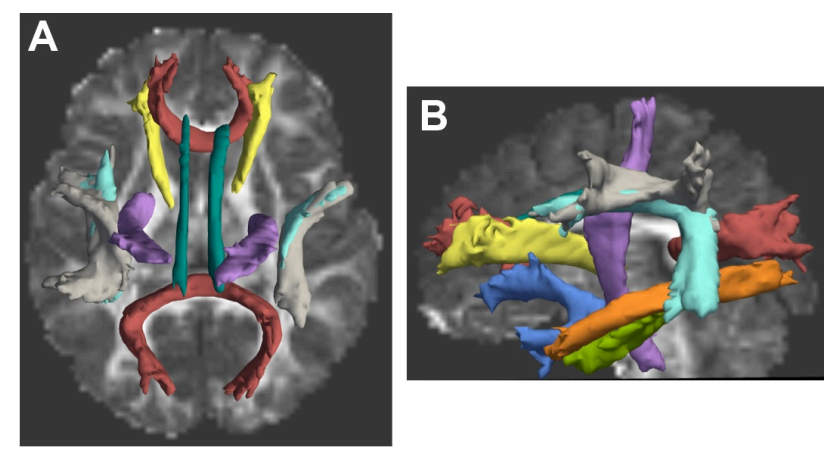

Figure I An example of reconstructed 18 white matter pathways: the corticospinal tract (violet), inferior longitudinal fasciculus (orange), uncinate fasciculus (blue), anterior thalamic radiation (yellow), cingulum cingulate gyrus (supracallosal) bundle (green), cingulum angular (infracallosal) bundle (CAB) (light green), superior longitudinal fasciculus parietal bundle (gray), superior longitudinal fasciculus temporal bundle (cyan), corpus callosum forceps major (FMaj) (red, posterior), and corpus callosum forceps minor (red, anterior).

Notes: Figures show an axial slice $(\mathbf{A})$ and a sagittal slice (B). 
visually and there were no failed tracts with aberrant trajectories. We adopted a weighted average value, as TRACULA is based upon probabilistic tractography.

\section{Statistical analyses}

Statistical analyses were performed using SPSS version 24.0 for Apple Mac. Betweengroup analyses involving age and years of education were conducted using an independent $t$-test. Gender was evaluated using the chi-squared test and handedness was assessed with Fisher's exact test. The statistical significance level was set at $p<0.05$. Since we aimed to assess which diffusivity measurements in white matter fibers can predict the diagnosis of OCD, we conducted logistic regression analysis. We entered FA, AD, MD, and $\mathrm{RD}$ of 18 tracts ( 72 variables) into the independent variables for the logistic regression. We used the forward selection method which selects the most significant of these variables and set at 0.05 . We also examined the interaction effect between selected variables. In addition, we conducted two analyses; we treated age as a covariate in one analysis and age, gender, and handedness as covariates in the other analysis to control the effect of these factors. We also drew scatter plots and calculated the Spearman's rank-order correlation between diffusivity measurements and clinical features (duration of illness, age of onset, and Y-BOCS score) of OCD.

\section{Results}

\section{Demographics and clinical characteristics}

We enrolled 25 patients with OCD and 27 healthy agematched controls. Age, gender, handedness, and years of education were not significantly different when compared between OCD and HC groups (Table 1). The mean and SD of

Table I Demographic and clinical characteristics

\begin{tabular}{llll}
\hline Variables & OCD, $\mathbf{n}=\mathbf{2 5}$ & $\mathbf{H C}, \mathbf{n}=\mathbf{2 7}$ & $\mathbf{p}$-value \\
\hline Age (years) & $35.0( \pm 8.9)$ & $32.4( \pm 6.7)$ & 0.235 \\
Gender (male/female) & $7 / 18$ & $13 / 14$ & 0.163 \\
Handedness (right/left) & $23 / 2$ & $26 / 1$ & 0.603 \\
Years of education & $14.3( \pm 2.2)$ & $15.4( \pm 2.0)$ & 0.062 \\
Age of onset (years) & $25.9( \pm 8.6)$ & $\mathrm{N} / \mathrm{A}$ & $\mathrm{N} / \mathrm{A}$ \\
Duration of illness (years) & $8.6( \pm 5.9)$ & $\mathrm{N} / \mathrm{A}$ & $\mathrm{N} / \mathrm{A}$ \\
Y-BOCS-total & $22.2( \pm 6.7)$ & $\mathrm{N} / \mathrm{A}$ & $\mathrm{N} / \mathrm{A}$ \\
Y-BOCS-obsessions & $11.0( \pm 3.1)$ & $\mathrm{N} / \mathrm{A}$ & $\mathrm{N} / \mathrm{A}$ \\
Y-BOCS-compulsions & $11.1( \pm 3.8)$ & $\mathrm{N} / \mathrm{A}$ & $\mathrm{N} / \mathrm{A}$ \\
HAM-D I7 & $3.8( \pm 3.4)$ & $\mathrm{N} / \mathrm{A}$ & $\mathrm{N} / \mathrm{A}$ \\
HAM-A & $5( \pm 4.5)$ & $\mathrm{N} / \mathrm{A}$ & $\mathrm{N} / \mathrm{A}$ \\
\hline
\end{tabular}

Note: Values represent the mean $( \pm S D)$.

Abbreviations: HAM-A, Hamilton Anxiety Rating Scale; HAM-D, Hamilton Depression Rating Scale; HC, healthy control; N/A, not applicable; OCD, obsessivecompulsive disorder; Y-BOCS, Yale-Brown Obsessive Compulsive Scale. duration of OCD, Y-BOCS score, HAM-D 17, and HAM-A are also given in Table 1.

\section{Integrity of the white matter tract integrity}

Logistic regression analysis revealed an increased RD in the FMaj $(B>0, p=0.020)$ and a reduction of $\mathrm{RD}$ in the right $\mathrm{CAB}(B<0, p=0.004)$ can predict the probability of OCD (Table 2). We showed the whole diffusivity mesurements in 18 white matter pathways in Table S1. The goodness-offit index of this model was 0.257 for Cox and Snell's $R^{2}$. Figure 2 shows the FMaj (red) and the right CAB (cyan). We also examined the interaction between $R D$ values within the FMaj and the right CAB; however, there were no significant indication of interaction. We also conducted analyses in which we used age as a covariate and age, gender, and handedness as covariates to adjust the effect of these factors. These indices were significant when we include only age as a covariate (Table 3A). However, when age, gender, and handedness were included as covariates, there were a significant increase of MD in FMaj and decrease of RD in right $\mathrm{CAB}$ (Table 3B).

\section{Correlation between changes in the white matter and clinical features}

A scatter plot and Spearman's rank-order correlation did not detect a significant correlation between $\mathrm{RD}$ values in the FMaj and clinical features (duration of illness, age of onset, and Y-BOCS score) and that between the right $\mathrm{CAB}$ and clinical characetristics (Figures S1 and S2).

\section{Discussion}

In this study, we used TRACULA which is an automated probabilistic tractography to reconstruct 18 major white matter tracts to detect alterations in the white matter microstructure of OCD patients. We found that the OCD group showed an increased $\mathrm{RD}$ in the FMaj and a reduced $\mathrm{RD}$ in the right $\mathrm{CAB}$.

DTI data provide four highly useful metrics: FA, RD, $\mathrm{AD}$, and MD. $\mathrm{RD}$ is equal to the average of the two smaller eigenvalues ${ }^{43}$ and is also considered to be a useful indicator for the evaluation of myelination; ${ }^{44}$ an increased RD is associated with the loss of myelination ${ }^{45}$ and reduced $\mathrm{RD}$ is attributed to strong coherence (eg, higher density, stronger myelination, and more coherent fibers) ${ }^{46}$ However, changes in $\mathrm{AD}$ are seen in axonal injury. ${ }^{47}$ Based on this, our present results reflect demyelination in the FMaj and more coherence such as increased coherence of the right $\mathrm{CAB}$. 
Table 2 RD values of white matter tracts which showed significance in logistic regression analysis

\begin{tabular}{|c|c|c|c|c|}
\hline \multirow[t]{2}{*}{ White matter tract } & \multicolumn{2}{|l|}{$\mathrm{RD}\left(\times 10^{-3} \mathrm{~mm}^{2} / \mathrm{s}\right)$} & \multirow[t]{2}{*}{ B } & \multirow[t]{2}{*}{$p$-value } \\
\hline & OCD & $\mathrm{HC}$ & & \\
\hline Forceps major & $0.5756(0.5437-0.6218)$ & $0.5382(0.4979-0.5910)$ & 14,083 & 0.020 \\
\hline rh. cingulum angular (infracallosal) bundle & $0.7557(0.7360-0.776 \mathrm{I})$ & $0.7838(0.7657-0.8031)$ & $-36,150$ & 0.004 \\
\hline
\end{tabular}

Notes: Values represent the median (interquartile range).

Abbreviations: $\mathrm{HC}$, healthy control; OCD, obsessive-compulsive disorder; RD, radial diffusivity; rh., right.

The FMaj connects the right and left occipital lobes through the splenium of corpus callosum. ${ }^{48}$ The increased RD observed in the FMaj is partly consistent with previous DWI studies. For example, Fan et al ${ }^{28}$ reported an increased RD in the left occipital lobe, but without a change in $\mathrm{AD}$, within non-medicated OCD patients. This suggests a loss of myelin integrity in this region. Similarly, an increased RD in the splenium of the corpus callosum was identified by Magioncalda et al, ${ }^{31}$ although $\mathrm{AD}$ was not the target of this particular investigation. Radua et al reported increased white matter volume and decreased FA in FMaj and increased white matter volume in cingulum angular bundle and FMaj. ${ }^{29}$ Patients with OCD have visuo-spatial dysfunction in Rey Complex Figure Test and Recognition Trial. ${ }^{49}$ Our findings suggest dysmyelination in FMaj, which connects occipital lobe to each other, might contribute to visuospatial impairment. This alteration in FMaj possibly contributes to the symptom induced by the visual stimulation (eg, some OCD patients feel contaminated

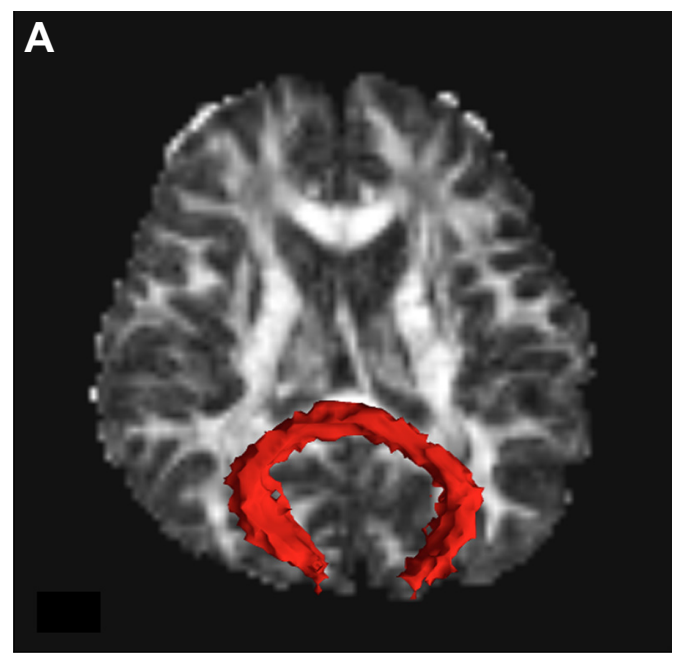

\section{B}
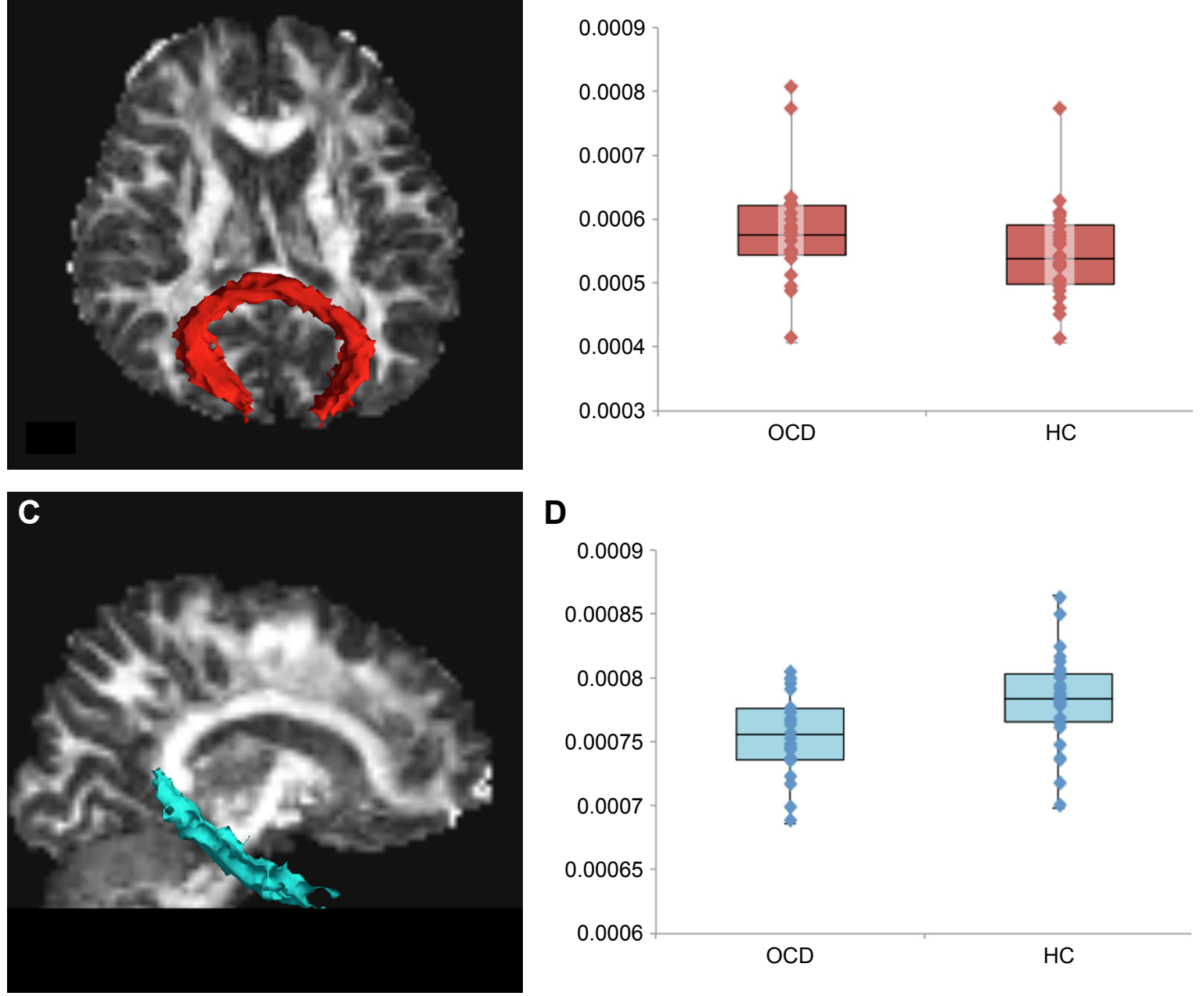

Figure 2 Reconstruction of the forceps major (FMaj, red) and the right cingulum-angular (infracallosal) bundle (CAB, cyan) in a representative OCD patient. Notes: Figures show an axial (A) and a sagittal slice (C). Box plots show RD values of the FMaj (B) and the right $C A B(D)$.

Abbreviations: $\mathrm{HC}$, healthy control; OCD, obsessive-compulsive disorder; RD, radial diffusivity. 
Table 3 Regression coefficient and $p$-values of two white matter tracts showing significance in Table 2 , including $(A)$ age and (B) age, gender, and handedness

\begin{tabular}{lll}
\hline Parameter in the tract & B & p-value \\
\hline (A) & & \\
\hline RD in forceps major $\left(\times 10^{-3} \mathrm{~mm}^{2} / \mathrm{s}\right)$ & 13,799 & 0.023 \\
RD in rh. cingulum angular (infracallosal) bundle & $-36,020$ & 0.005 \\
$\left(\times 10^{-3} \mathrm{~mm}^{2} / \mathrm{s}\right)$ & & \\
\hline (B) & & \\
\hline $\mathrm{MD}$ in forceps major $\left(\times 10^{-3} \mathrm{~mm}^{2} / \mathrm{s}\right)$ & 17,844 & 0.040 \\
RD in rh. cingulum angular (infracallosal) bundle & $-43,631$ & 0.006 \\
$\left(\times 10^{-3} \mathrm{~mm}^{2} / \mathrm{s}\right)$ & & \\
\hline
\end{tabular}

Abbreviations: MD, mean diffusivity; rh., right; RD, radial diffusivity.

though they touch nothing, other OCD patients symmetrically arrange their belongings). Recent researches including a systematic review and meta-analysis suggested that the white matter tracts in the cortico-striato-thalamo-cortical loop and the intra-hemispheric bundles (ie, inferior fronto-occipital fasciculus, superior longitudinal fasciculus, inferior longitudinal fasciculus, and uncinate fasciculus), had microstructural abnormalities in OCD patients. ${ }^{7,50}$ Nevertheless, our current findings suggest that posterior inter-hemispheric bundles (ie, the FMaj) are also involved in the neuropathology of OCD. In the current study, we found an increased RD in FMaj in the OCD group when we used only age as a covariate. However, when age, gender, and handedness were entered as covariates, the OCD group showed higher MD value in the same white matter tract. An increase in RD (the average of the two small eigenvalues) leads to an increase in MD (the average of the tensor's eigenvalues). Therefore, we consider that we might identify some alteration in the FMaj. Gan et al examined diffusivity measurements with TBSS and reported FA reduction in the splenium, genu, and body as well as increased RD in the genu and body of the corpus callosum in OCD patients. ${ }^{22}$ Since TRACULA only reconstructed the FMaj and minor, the current study was unable to show data on the body. They found lower FA while we observed higher $\mathrm{RD}$ in the splenium, thus the decreased FA in their study might be due to dysmyelination. In our study, there was no $\mathrm{RD}$ alteration in the genu where forceps minor traverses. It is possible that medication status was related to this because Benedetti et al reported differences in RD values in the genu between medicated and drug-naive patients. ${ }^{30}$

Most previous studies used the "cingulum bundle" as a single white matter tract. However, TRACULA defined the cingulum as two separate segments; the cingulate gyrus (supracallosal) bundle and the angular (infracallosal) bundle (CAB).$^{33}$ The former is the superior part of the cingulum and runs along the cingulate gyrus, while the latter, the $\mathrm{CAB}$, is the inferior part and exists along the ventral hippocampus. ${ }^{48}$ The $\mathrm{CAB}$ projects into the parahippocampal gyrus and terminates in the medial temporal lobe. ${ }^{51}$ We identified a lower $\mathrm{RD}$ in the (right) $\mathrm{CAB}$ and Fan et al previously reported a similar change in the same part of cingulum. ${ }^{52}$ The cingulum consists of many fibers of various lengths and the longest fibers connect the anterior temporal gyrus and the orbitofrontal cortex. ${ }^{51-53}$ Since cingulotomy, targeting the superior anterior cingulum, is an effective treatment for $\mathrm{OCD},{ }^{54}$ we can hypothesize that cingulotomy may also disrupt the strong coherence of the long fibers, which run both anterior cingulum and $\mathrm{CAB}$. Concerning parahippocampal gyrus, previous studies reported volume change in the parahippocampal gyrus among OCD patients. ${ }^{8,44,55,56}$ It is a part of limbic system and the region was activated in the aversion in OCD patients ${ }^{57}$ and $\mathrm{MD}$ increase in the area was showed by Spalletta et al. ${ }^{50}$ The alteration in the $\mathrm{CAB}$ might be associated with excessive representation of aversive stimuli.

Our results did not show the correlation between diffusivity measurements and clinical features. Some of the past studies did not show the correlations between $\mathrm{RD}$ values in each area, which showed significance when compared with $\mathrm{HC}^{20,28,31}$ and these were consistent with our results.

In OCD pathology, myelin status might play a key role. Glahn et al used magnetization transfer imaging, which is sensitive to subtle neuropathological changes such as myelination. ${ }^{58}$ In their study, patients with OCD showed an increase of magnetization transfer ratio in the left middle frontal gyrus and gray matter of the right inferior frontal gyrus, which could be associated with myelination.

Recent published study, which used a similar method as ours, reported significant FA decrease in the FMaj and the right uncinate fasciculus. ${ }^{59}$ These data were obtained from various races and all of our data were obtained from Asians. An initial study showed racial differences in MD for gray matter integrity. ${ }^{60}$ They did not detect racial differences in white matter hyperintensities but our results might implicate racial differences in white matter integrity.

Since previous studies have reported that diffusion parameters might be affected by age, gender, and handedness, ${ }^{61-63}$ we included these parameters as covariates. Increased RD in the FMaj and decreased $\mathrm{RD}$ in the right $\mathrm{CAB}$ may contribute to the probability of OCD; this was especially notable even after we considered the influence of demographic characteristics.

There are several limitations in this study. First, we obtained the weighted averages over the entire pathways 
using TRACULA and those values were not focal. TRACULA can identify the specific tract and TBSS can detect the focal abnormality, and so further studies which combined these methods would overcome each limitation. Another limitation is that TRACULA is unable to reconstruct the corpus callosum, except for the FMaj and minor. Therefore, we could not refer to white matter alterations around the body of the corpus callosum. In addition, other tracts also were not considered. For example, it does not construct the cortico-ponto-cerebellar tract, so we could not identify the alteration associated with cerebellum.

\section{Conclusion}

We found white matter alterations, increased RD in the FMaj which is the occipital inter-hemisphere fibers, and in the right angular (infracallosal) bundle which connects the medial temporal lobe and other regions in patients with OCD. These results suggest that the pathogenesis of OCD may include changes in myelination in not only the fronto-striato-thalamic circuit but also the posterior and temporal regions.

\section{Acknowledgments}

We thank T Okada, H Ito, and technical engineers for their assistance in MRI data acquisition. This work was partly supported by the "Application of DecNef for development of diagnostic and cure system for mental disorders and construction of clinical application bases" of the SRPBS from MEXT and AMED, JSPS KAKENHI Grant Numbers 25119001, 26120721 and 16K19778, and the Joint Usage/ Research Center at ISER, Osaka University.

\section{Author contributions}

All authors contributed toward data analysis, drafting and revising the paper and agree to be accountable for all aspects of the work.

\section{Disclosure}

The authors report no conflicts of interest in this work.

\section{References}

1. Ruscio AM, Stein DJ, Chiu WT, Kessler RC. The epidemiology of obsessive-compulsive disorder in the National Comorbidity Survey Replication. Mol Psychiatry. 2010;15(1):53-63.

2. Michaud CM, McKenna MT, Begg S, et al. The burden of disease and injury in the United States 1996. Popul Health Metr. 2006;4:11.

3. Huppert JD, Simpson HB, Nissenson KJ, Liebowitz MR, Foa EB. Quality of life and functional impairment in obsessive-compulsive disorder: a comparison of patients with and without comorbidity, patients in remission, and healthy controls. Depress Anxiety. 2009;26(1):39-45.

4. Baxter LRJ, Schwartz JM, Mazziotta JC, et al. Cerebral glucose metabolic rates in nondepressed patients with obsessive-compulsive disorder. $\mathrm{Am}$ J Psychiatry. 1988;145(12):1560-1563.
5. Atmaca M, Yildirim H, Ozdemir H, Tezcan E, Poyraz AK. Volumetric MRI study of key brain regions implicated in obsessive-compulsive disorder. Prog Neuropsychopharmacol Biol Psychiatry. 2007;31(1): 46-52.

6. Menzies L, Chamberlain SR, Laird AR, Thelen SM, Sahakian BJ, Bullmore ET. Integrating evidence from neuroimaging and neuropsychological studies of obsessive-compulsive disorder: the orbitofrontostriatal model revisited. Neurosci Biobehav Rev. 2008;32(3):525-549.

7. Piras F, Piras F, Caltagirone C, Spalletta G. Brain circuitries of obsessive compulsive disorder: a systematic review and meta-analysis of diffusion tensor imaging studies. Neurosci Biobehav Rev. 2013;37(10 Pt 2): 2856-2877.

8. de Wit SJ, Alonso P, Schweren L, et al. Multicenter voxel-based morphometry mega-analysis of structural brain scans in obsessivecompulsive disorder. Am J Psychiatry. 2014;171(3):1-10.

9. Piras F, Piras F, Chiapponi C, Girardi P, Caltagirone C, Spalletta G. Widespread structural brain changes in OCD: a systematic review of voxel-based morphometry studies. Cortex. 2015;62:89-108.

10. Zai G, Bezchlibnyk YB, Richter MA, et al. Myelin oligodendrocyte glycoprotein (MOG) gene is associated with obsessive-compulsive disorder. Am J Med Genet B Neuropsychiatr Genet. 2004;129B(1):64-68.

11. Stewart SE, Platko J, Fagerness J, et al. A genetic family-based association study of OLIG2 in obsessive-compulsive disorder. Arch Gen Psychiatry. 2007;64(2):209-214.

12. Atmaca M, Onalan E, Yildirim H, Yuce H, Koc M, Korkmaz S. The association of myelin oligodendrocyte glycoprotein gene and white matter volume in obsessive-compulsive disorder. J Affect Disord. 2010;124(3):309-313.

13. Yoo SY, Jang JH, Shin YW, et al. White matter abnormalities in drugnaïve patients with obsessive-compulsive disorder: a Diffusion Tensor Study before and after citalopram treatment. Acta Psychiatr Scand. 2007;116(3):211-219.

14. Saito Y, Nobuhara K, Okugawa G, et al. Corpus callosum in patients with obsessive-compulsive disorder: diffusion-tensor imaging study. Radiology. 2008;246(2):536-542.

15. Garibotto V, Scifo P, Gorini A, et al. Disorganization of anatomical connectivity in obsessive compulsive disorder: a multi-parameter diffusion tensor imaging study in a subpopulation of patients. Neurobiol Dis. 2010;37(2):468-476.

16. Bora E, Harrison BJ, Fornito A, et al. White matter microstructure in patients with obsessive-compulsive disorder. $J$ Psychiatry Neurosci. 2011;36(1):42-46.

17. Li F, Huang X, Yang Y, et al. Microstructural brain abnormalities in patients with obsessive-compulsive disorder: diffusion-tensor MR imaging study at 3.0 T. Radiology. 2011;260(1):216-223.

18. Nakamae T, Narumoto J, Sakai Y, et al. Diffusion tensor imaging and tract-based spatial statistics in obsessive-compulsive disorder. J Psychiatr Res. 2011;45(5):687-690.

19. Zarei M, Mataix-Cols D, Heyman I, et al. Changes in gray matter volume and white matter microstructure in adolescents with obsessivecompulsive disorder. Biol Psychiatry. 2011;70(11):1083-1090.

20. Jayarajan RN, Venkatasubramanian G, Viswanath B, et al. White matter abnormalities in children and adolescents with obsessive-compulsive disorder: a diffusion tensor imaging study. Depress Anxiety. 2012; 29(9):780-788.

21. Oh JS, Jang JH, Jung WH, et al. Reduced fronto-callosal fiber integrity in unmedicated OCD patients: a diffusion tractography study. Hum Brain Mapp. 2012;33(10):2441-2452.

22. Gan J, Zhong M, Fan J, et al. Abnormal white matter structural connectivity in adults with obsessive-compulsive disorder. Transl Psychiatry. 2017;7(3):e1062.

23. Szeszko PR, Ardekani BA, Ashtari M, et al. White matter abnormalities in obsessive-compulsive disorder: a diffusion tensor imaging study Arch Gen Psychiatry. 2005;62(7):782-790.

24. Cannistraro PA, Makris N, Howard JD, et al. A diffusion tensor imaging study of white matter in obsessive-compulsive disorder. Depress Anxiety. 2007;24(6):440-446. 
25. Ha TH, Kang DH, Park JS, et al. White matter alterations in male patients with obsessive-compulsive disorder. Neuroreport. 2009; 20(7):735-739.

26. Lochner C, Fouche JP, du Plessis S, et al. Evidence for fractional anisotropy and mean diffusivity white matter abnormalities in the internal capsule and cingulum in patients with obsessive-compulsive disorder. J Psychiatry Neurosci. 2012;37(3):193-199.

27. Peng Z, Lui SS, Cheung EF, et al. Brain structural abnormalities in obsessive-compulsive disorder: converging evidence from white matter and grey matter. Asian J Psychiatr. 2012;5(4):290-296.

28. Fan Q, Yan X, Wang J, et al. Abnormalities of white matter microstructure in unmedicated obsessive-compulsive disorder and changes after medication. PLoS One. 2012;7(4):e35889.

29. Radua J, Grau M, van den Heuvel OA, et al. Multimodal voxel-based meta-analysis of white matter abnormalities in obsessive-compulsive disorder. Neuropsychopharmacology. 2014;39(7):1547-1557.

30. Benedetti F, Giacosa C, Radaelli D, et al. Widespread changes of white matter microstructure in obsessive-compulsive disorder: effect of drug status. Eur Neuropsychopharmacol. 2013;23(7):581-593.

31. Magioncalda P, Martino M, Ely BA, Inglese M, Stern ER. Microstructural white-matter abnormalities and their relationship with cognitive dysfunction in obsessive-compulsive disorder. Brain Behav. 2016; 6(3):e00442.

32. O'Donnell LJ, Pasternak O. Does diffusion MRI tell us anything about the white matter? An overview of methods and pitfalls. Schizophr Res. 2015;161(1):133-141.

33. Yendiki A, Panneck P, Srinivasan P, et al. Automated probabilistic reconstruction of white-matter pathways in health and disease using an atlas of the underlying anatomy. Front Neuroinform. 2011;5:23.

34. Chiu CH, Lo YC, Tang HS, et al. White matter abnormalities of frontostriato-thalamic circuitry in obsessive-compulsive disorder: a study using diffusion spectrum imaging tractography. Psychiatry Res. 2011; 192(3):176-182.

35. Nakamae T, Sakai Y, Abe Y, et al. Altered fronto-striatal fiber topography and connectivity in obsessive-compulsive disorder. PLoS One. 2014;9(11):e112075.

36. Fischl B, Salat DH, Busa E, et al. Whole brain segmentation: automated labeling of neuroanatomical structures in the human brain. Neuron. 2002;33(3):341-355.

37. Yeatman JD, Dougherty RF, Myall NJ, Wandell BA, Feldman HM. Tract profiles of white matter properties: automating fiber-tract quantification. PLoS One. 2012;7(11):e49790.

38. First MB. User's Guide for the Structured Clinical Interview for DSM-IV Axis I Disorders SCID-I: Clinician Version. Washington, DC: American Psychiatric Press; 1997.

39. Nakajima T, Nakamura M, Taga C, et al. Reliability and validity of the Japanese version of the Yale-Brown Obsessive-Compulsive Scale. Psychiatry Clin Neurosci. 1995;49(2):121-126.

40. Desikan RS, Segonne F, Fischl B, et al. An automated labeling system for subdividing the human cerebral cortex on MRI scans into gyral based regions of interest. Neuroimage. 2006;31(3):968-980.

41. Yendiki A, Koldewyn K, Kakunoori S, Kanwisher N, Fischl B. Spurious group differences due to head motion in a diffusion MRI study. Neuroimage. 2014;88:79-90.

42. Behrens TE, Berg HJ, Jbabdi S, Rushworth MF, Woolrich MW. Probabilistic diffusion tractography with multiple fibre orientations: what can we gain? Neuroimage. 2007;34(1):144-155.

43. O'Donnell LJ, Westin CF. An introduction to diffusion tensor image analysis. Neurosurg Clin N Am. 2011;22(2):185-196, viii.

44. Alger JR. The diffusion tensor imaging toolbox. J Neurosci. 2012; 32(22):7418-7428.

45. Song S-K, Sun S-W, Ramsbottom MJ, Chang C, Russell J, Cross AH. Dysmyelination revealed through MRI as increased radial (but unchanged axial) diffusion of water. Neuroimage. 2002;17(3): $1429-1436$.
46. Jones DK, Knosche TR, Turner R. White matter integrity, fiber count, and other fallacies: the do's and don'ts of diffusion MRI. Neuroimage. 2013;73:239-254.

47. Song SK, Sun SW, Ju WK, Lin SJ, Cross AH, Neufeld AH. Diffusion tensor imaging detects and differentiates axon and myelin degeneration in mouse optic nerve after retinal ischemia. Neuroimage. 2003;20(3): 1714-1722.

48. Wakana S, Caprihan A, Panzenboeck MM, et al. Reproducibility of quantitative tractography methods applied to cerebral white matter. Neuroimage. 2007;36(3):630-644.

49. Savage CR, Baer L, Keuthen NJ, Brown HD, Rauch SL, Jenike MA. Organizational strategies mediate nonverbal memory impairment in obsessive-compulsive disorder. Biol Psychiatry. 1999;45(7):905-916.

50. Spalletta G, Piras F, Fagioli S, Caltagirone C, Piras F. Brain microstructural changes and cognitive correlates in patients with pure obsessive compulsive disorder. Brain Behav. 2014;4(2):261-277.

51. Catani M, Thiebaut de Schotten M. A diffusion tensor imaging tractography atlas for virtual in vivo dissections. Cortex. 2008;44(8): 1105-1132.

52. Fan S, van den Heuvel OA, Cath DC, et al. Mild white matter changes in un-medicated obsessive-compulsive disorder patients and their unaffected siblings. Front Neurosci. 2015;9:495.

53. Jones DK, Christiansen KF, Chapman RJ, Aggleton JP. Distinct subdivisions of the cingulum bundle revealed by diffusion MRI fibre tracking: implications for neuropsychological investigations. Neuropsychologia. 2013;51(1):67-78.

54. Greenberg BD, Rauch SL, Haber SN. Invasive circuitry-based neurotherapeutics: stereotactic ablation and deep brain stimulation for OCD. Neuropsychopharmacology. 2010;35(1):317-336.

55. Valente AA Jr, Miguel EC, Castro CC, et al. Regional gray matter abnormalities in obsessive-compulsive disorder: a voxel-based morphometry study. Biol Psychiatry. 2005;58(6):479-487.

56. Tang W, Huang X, Li B, et al. Structural brain abnormalities correlate with clinical features in patients with drug-naive OCD: a DARTELenhanced voxel-based morphometry study. Behav Brain Res. 2015; 294:72-80.

57. Mataix-Cols D, Cullen S, Lange K, et al. Neural correlates of anxiety associated with obsessive-compulsive symptom dimensions in normal volunteers. Biol Psychiatry. 2003;53(6):482-493.

58. Glahn A, Prell T, Grosskreutz J, Peschel T, Muller-Vahl KR. Obsessivecompulsive disorder is a heterogeneous disorder: evidence from diffusion tensor imaging and magnetization transfer imaging. $B M C$ Psychiatry. 2015;15:135.

59. He X, Steinberg E, Stefan M, Fontaine M, Simpson HB, Marsh R. Altered frontal interhemispheric and fronto-limbic structural connectivity in unmedicated adults with obsessive-compulsive disorder. Hum Brain Mapp. 2018;39(2):803-810.

60. Liu G, Allen B, Lopez O, et al. Racial differences in gray matter integrity by diffusion tensor in black and white octogenarians. Curr Alzheimer Res. 2015;12(7):648-654.

61. Hsu JL, Leemans A, Bai CH, et al. Gender differences and age-related white matter changes of the human brain: a diffusion tensor imaging study. Neuroimage. 2008;39(2):566-577.

62. Lebel C, Gee M, Camicioli R, Wieler M, Martin W, Beaulieu C. Diffusion tensor imaging of white matter tract evolution over the lifespan. Neuroimage. 2012;60(1):340-352.

63. Powell JL, Parkes L, Kemp GJ, Sluming V, Barrick TR, Garcia-Finana M. The effect of sex and handedness on white matter anisotropy: a diffusion tensor magnetic resonance imaging study. Neuroscience. 2012; 207:227-242. 


\section{Publish your work in this journal}

Neuropsychiatric Disease and Treatment is an international, peerreviewed journal of clinical therapeutics and pharmacology focusing on concise rapid reporting of clinical or pre-clinical studies on a range of neuropsychiatric and neurological disorders. This journal is indexed on PubMed Central, the 'PsycINFO' database and CAS, and is the official journal of The International Neuropsychiatric Association (INA). The manuscript management system is completely online and includes a very quick and fair peer-review system, which is all easy to use. Visit http://www.dovepress.com/testimonials.php to read real quotes from published authors.

\footnotetext{
Submit your manuscript here: http://www.dovepress.com/neuropsychiatric-disease-and-treatment-journal
} 